\title{
Permeability through Cellulose Membranes Grafted with Vinyl Monomers in Homogeneous System II. States of Water in Acrylonitrile Grafted Cellulose Membranes
}

\author{
Noboru Nishioka, Seiichiro Yoshimi, Tatsuya IwAGUCHI, \\ and Kouichi KOSAI \\ Faculty of Engineering, Osaka Electro-Communication University, \\ Neyagawa, Osaka 572, Japan
}

(Received April 3, 1984)

\begin{abstract}
Differential scanning calorimetry was used to investigate the states of water in cellulose membranes grafted with acrylonitrile (AN). Examination of the melting endotherms and freezing exotherms showed the existence of three water states differing in their interaction with polymer molecules: free water, intermediate water, and bound water. The amount of intermediate water was remarkably reduced by grafting AN onto cellulose. The amounts of freezing water and nonfreezing water were estimated to elucidate the diffusive permeability of solutes through the membranes. The difference in permeability through the membranes may be explained by the difference in freezing water content. The intermediate water may not be appreciably related to the permeation of solutes through the membranes.
\end{abstract}

KEY WORDS Cellulose / Acrylonitrile / Grafted Membrane / Differential Scanning Calorimetry / States of Water / Free Water / Intermediate Water / Bound Water /

A number of terms have been used to describe the various states of water molecules in waterswollen membranes. ${ }^{1}$ Some water molecules are bound to polymer molecules through hydrogen bonding and are immobilized and thus referred to as "bound water." Other water molecules do not take part in hydrogen bonding with polymer molecules and thus have a greater degree of mobility and are referred to as "free water." ${ }^{-9}$ Still, other water molecules have been reported to interact weakly with polymer molecules and are thus referred to as "intermediate water" or "secondary bound water."1,10-20 Among them, bound water is thought to be less capable of dissolving solutes because of its lower mobility and dielectric constant. ${ }^{21,22}$ Characterization of water in regard to the diffusive permeation of solutes, thus, seems highly important for clarifying the mechanism of membrane perm- selectivity.

A number of experimental techniques have been used to investigate the states of water in membranes such as differential scanning calorimetry (DSC), ${ }^{1,7-9,15-20}$ neuclear magnetic resonance, ${ }^{2-6,11,12,19}$ dilatometry, ${ }^{10,13,14}$ specific conductivity, ${ }^{13,14}$ and dielectric relaxation. ${ }^{14}$ The investigation of the freezing behavior of water in membranes has indicated the existence of nonfreezing water which does not freeze even when cooled down far below the freezing point. $^{7}$ It is considered that the nonfreezing water is bound water and the other, free water and intermediate water. DSC makes possible the quantitative determination of the amounts of nonfreezing and freezing water.

In our previous paper, ${ }^{23}$ the diffusive permeability of solutes through cellulose membranes grafted with acrylonitrile (AN) was 
Table I. Characterization of grafted and blended membranes ${ }^{\mathrm{a}}$

\begin{tabular}{|c|c|c|c|c|}
\hline \multirow{2}{*}{ Sample code } & \multirow{2}{*}{$\begin{array}{c}\begin{array}{c}\text { Grafting } \\
\text { efficiency }\end{array} \\
\%\end{array}$} & \multicolumn{3}{|c|}{ PAN content $/ \mathrm{wt} \%$} \\
\hline & & Overall & Graft-PAN & Homo-PAN \\
\hline \multicolumn{5}{|l|}{ H-GE Membrane } \\
\hline g-PAN 1 & 74.9 & 12.6 & 10.0 & 2.6 \\
\hline g-PAN 2 & 90.8 & 33.9 & 30.8 & 3.1 \\
\hline \multicolumn{5}{|l|}{ L-GE Membrane } \\
\hline g-PAN 11 & 11.0 & 17.9 & 2.0 & 15.9 \\
\hline g-PAN 12 & 10.0 & 26.1 & 2.6 & 23.5 \\
\hline g-PAN 13 & 10.5 & 38.9 & 4.0 & 34.9 \\
\hline g-PAN 14 & 11.0 & 61.9 & 6.8 & 55.1 \\
\hline \multicolumn{5}{|c|}{ Blended membrane } \\
\hline b-PAN 1 & 0 & 10.0 & 0 & 10.0 \\
\hline b-PAN 3 & 0 & 30.0 & 0 & 30.0 \\
\hline b-PAN 5 & 0 & 50.0 & 0 & 50.0 \\
\hline
\end{tabular}

${ }^{a}$ Further details are given in ref 23.

investigated. Permeability was found to depend remarkably on the kinds of membranes such as higher grafting efficiency (H-GE membrane), lower grafting efficiency (L-GE membrane), and blended membranes. Permeability through the H-GE membranes was superior to that through the membrane cast from a dimethyl sulfoxide solution of cellulose, designated as the cellulose membrane. The good permeability through the H-GE membranes could not be explained by the difference in the water content of the membranes. In this work, the states of water in AN grafted cellulose membranes is discussed in relation to membrane permeability.

\section{EXPERIMENTAL}

\section{Materials}

The membranes prepared in our previous work $^{23}$ were used for this study. Table I summarizes the samples used. A Cuprophan membrane, commercial regenerated cellulose membrane produced by the cuprammonium process, and the cellulose membrane were compared with those grafted.

\section{DSC Measurements}

The membranes were immersed in distilled water for several days, wiped with paper to remove remaining surface water and sealed in aluminum pans. Each sample weight was about $10-20 \mathrm{mg}$. The samples were cooled with liquid nitrogen at a rate of $5 \mathrm{~K} \mathrm{~min}^{-1}$ to $-80^{\circ} \mathrm{C}$ and then heated at the same rate to $60-90^{\circ} \mathrm{C}$. The total water content was varied by standing the sample pans in the DSC cell at a given temperature for a given period to evaporate the water from the membranes. Measurements were repeated until the endothermic peaks disappeared. A differential scanning calorimeter, SEIKO SSC/560S DSC, was used. It was calibrated with indium as the standard. The heat of melting of the freezing water was determined from the area under the endothermic curve. The water content was expressed as the ratio of the weight of water in the membrane to that of the dry membrane and determined from the weight loss after drying the membrane at about $100^{\circ} \mathrm{C}$ to constant weight.

\section{RESULTS AND DISCUSSION}

The molecular weight dependence of diffusive permeability for several membranes is shown in Figure 1. Other details appear in the 
previous paper. ${ }^{23}$

The melting and freezing thermograms of the Cuprophan membrane are shown in Figure 2. The total water content was varied so as to investigate its influence on the states of water in the membrane. Although the sample pans were not sealed hermetically to permit water evaporation, the base lines of the melting

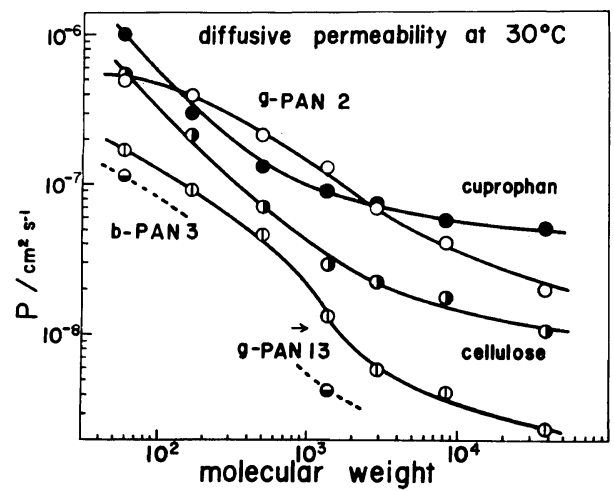

Figure 1. Molecular weight dependence of diffusive permeability for various membranes: (O) Cuprophan membrane; (O) cellulose membrane; (O) g-PAN 2 membrane; (1) g-PAN 13 membrane; ( $($ ) b-PAN 3 membrane; The solutes are, in the order of increasing molecular weight, $\mathrm{NaCl}$, uric acid, raffinose, vitamine $\mathbf{B}_{12}$, and three poly(ethylene glycol) of different molecular weights taken from the data cited in ref 23 . thermograms were stable up to about $20^{\circ} \mathrm{C}$, indicating no influence of water evaporation within the temperature range indicated. Several peaks are found on both thermograms, revealing the existence of several states of freezing water. A comparison with the results for pure water indicates that, on both thermograms, the peaks at higher temperatures may be assigned to the free water. The peaks at lower temperatures may be assigned to the water interacting weakly with polymer molecules, i.e., the intermediate water. It should be noted that even in the intermediate water, there may exist water differing in its interaction with polymer molecules, that is, relatively weakly interacting and relatively strongly interacting water. ${ }^{1,15}$

On the freezing thermograms, the free water peak at around $-20^{\circ} \mathrm{C}$ decreases sharply with decreasing water content and almost disappears at about 0.42 water content, whereas the peak for relatively strongly interacting water at around $-50^{\circ} \mathrm{C}$ hardly decreases at all, remaining generally at 0.42 . But, on the melting thermograms, the free water peak at around $0^{\circ} \mathrm{C}$ and the relatively weakly interacting water peak at around $-5^{\circ} \mathrm{C}$ decrease

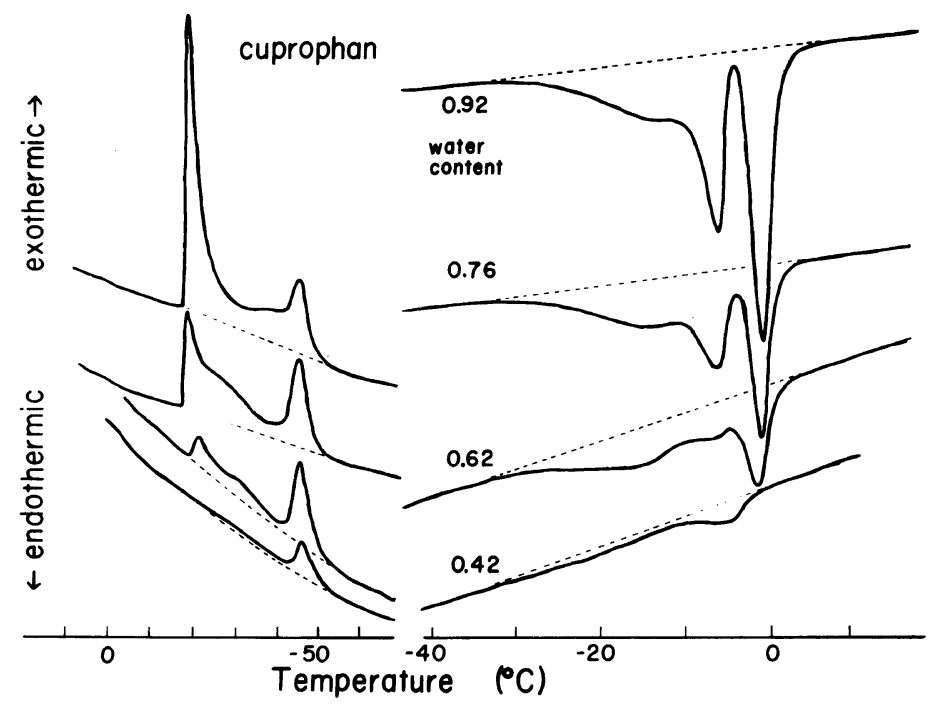

Figure 2. DSC freezing and melting thermograms for the Cuprophan membranes of various water content. 


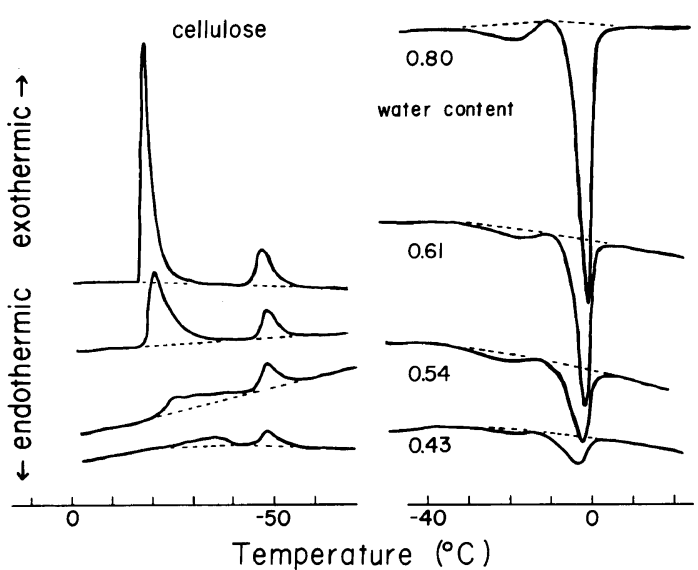

Figure 3. DSC freezing and melting thermograms for the cellulose membranes of various water content.

sharply with decreasing water content but the broad peak at around $-20^{\circ} \mathrm{C}$ decreases gradually. Moreover, in Figure 2, the free water peak shifts toward lower temperatures with decreasing water content but the relatively strongly interacting water peak shifts very little. These results indicate that the free water and relatively weakly interacting water decrease faster than the relatively strongly interacting water with decreasing total water content. ${ }^{15,16,18}$

Figure 3 shows the DSC thermograms of the cellulose membrane containing different amounts of water. The peaks corresponding to the free water at higher temperatures and intermediate water at lower temperatures can also be observed. But the disappearance of the sharp endothermic peak at around $-5^{\circ} \mathrm{C}$ and the broad exothermic peak between -50 and $-20^{\circ} \mathrm{C}$ indicates that the fraction of intermediate water in the cellulose membrane is smaller than that in the Cuprophan membrane.

As indicated above, differences in membrane preparation process influenced the states of water in membranes remarkably. The incorporation of polyacrylonitrile (PAN), a hydrophobic polymer, should thus influence the states of water in membranes even more sig-

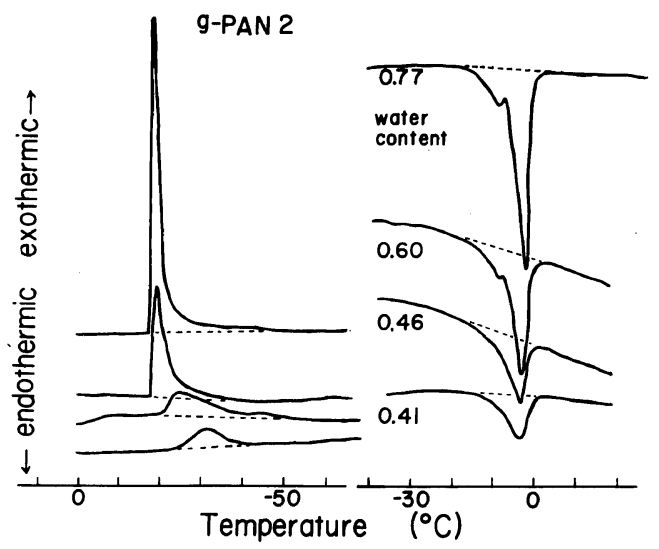

Figure 4. DSC freezing and melting thermograms for the g-PAN 2 membranes of various water content.

nificantly. It is well known that graft copolymers exhibit microphase separated structures. ${ }^{24}$ As indicated in the previous paper, ${ }^{23}$ in the case of our grafted membranes, the grafting efficiency affected the microphase separated structures remarkably. The DSC thermograms of the g-PAN 2 membrane, an H-GE membrane, are shown in Figure 4. The exothermic peak at around $-50^{\circ} \mathrm{C}$ and the endothermic peak at around $-20^{\circ} \mathrm{C}$ disappear with the appearance of the hump at around $-10^{\circ} \mathrm{C}$ on the melting endotherms, indicating a decrease in the relatively strongly interacting water. That is, the fraction of the relatively weakly interacting and/or free water to the total freezing water increased by grafting AN onto cellulose. This may qualitatively explain the good diffusive permeability through the g-PAN 2 membrane shown in Figure 1.

Figure 5 shows the DSC thermograms of the g-PAN 13 membrane, an L-GE membrane. The decrease in the relatively strongly interacting water is also evident but is not so remarkable as the g-PAN 2 membrane. However, the tailing of exothermic peaks at around $-20^{\circ} \mathrm{C}$ indicates an increase in the relatively weakly interacting water. A similar trend can also be seen in Figure 6 showing the DSC thermo- 


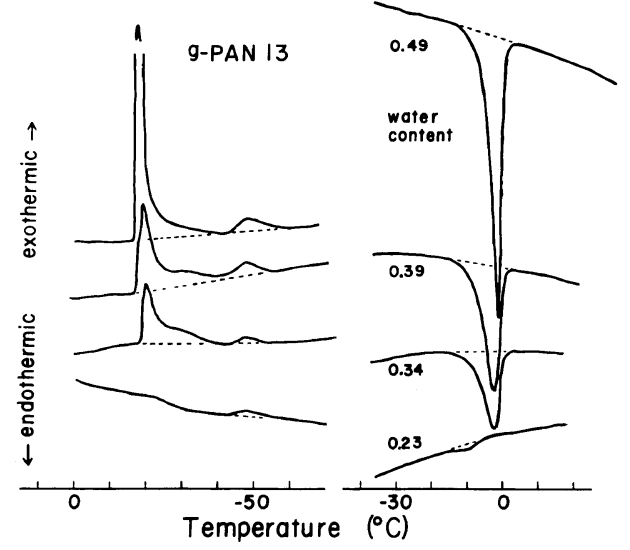

Figure 5. DSC freezing and melting thermograms for the g-PAN 13 membranes of various water content.

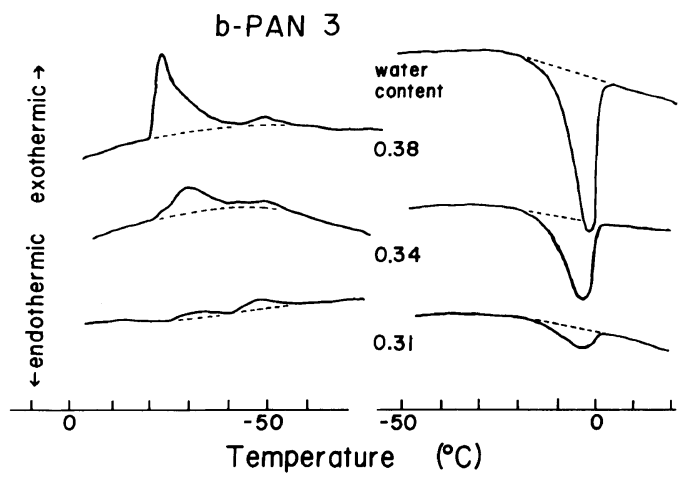

Figure 6. DSC freezing and melting thermograms for the b-PAN 3 membranes of various water content.

grams of the b-PAN 3 membrane, one of the blended membranes. It is of interest that the peaks for the relatively strongly interacting water appear on the freezing but not the melting thermograms. A similar tendency has been noted for the cellulose membranes grafted with other vinyl monomers, ${ }^{25}$ and thus, representation of both thermograms may be necessary to understand more precisely the states of water in membranes. Little work has been carried out on both thermograms. ${ }^{1}$

In consideration of diffusive permeation of solutes through water-swollen membranes, it is generally considered that permeating solutes can diffuse only through water phases in membranes. Yasuda et al..$^{26-28}$ showed that the

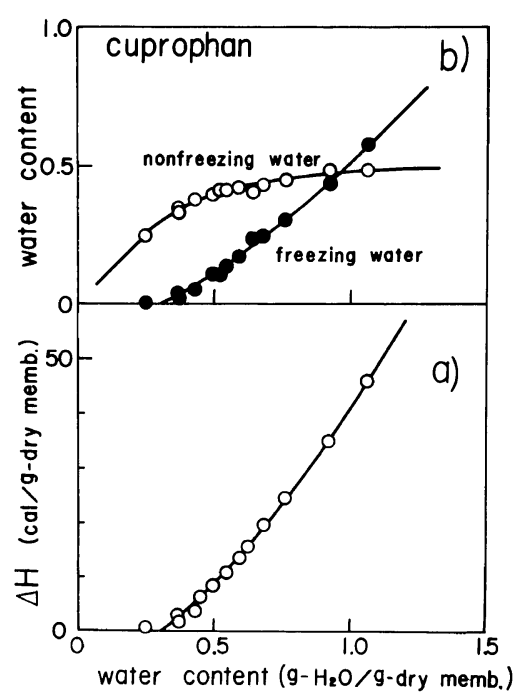

Figure 7. Total water content dependence of (a) the heat of melting of freezing water, $\Delta H$, and (b) nonfreezing and freezing water content for the Cuprophan membranes: $(\bigcirc)$ nonfreezing water; $(\bigcirc)$ freezing water.

permeability of solutes through membranes can be explained on the basis of water content. As can be seen from Figure 1, permeability through the g-PAN 2 membrane is superior to that through the cellulose membrane, whereas the water content of the g-PAN 2 membrane was nearly equal to that of the cellulose membrane. ${ }^{23}$ Water in membranes includes the nonfreezing water not thought to be appreciably related to the permeation of solutes. $^{21,22}$ Thus, the water related to the permeation of solutes should be considered.

Figure $7 \mathrm{a}$ shows the relationship between water content and the heat of melting of freezing water, $\Delta H$, for the Cuprophan membrane. Assuming the amount of nonfreezing water to be constant and the specific heat of melting of free water equal to that of intermediate water, plots of $\Delta H$ versus water content should fall on a straight line. The specific heat of melting of freezing water and the nonfreezing water content can be estimated from the slope of this straight line and the intercept of water content axis, respectively. But instead of a straight line, the curve con- 
vexed downward. It has generally been reported $^{8,9,16,17,20}$ that, except in certain cases, ${ }^{19,29}$ the specific heat of melting of freezing water is equal to that of pure water and thus the results shown in Figure $7 \mathrm{a}$ indicate that the amount of nonfreezing water varies with total water content.

Figure $7 \mathrm{~b}$ shows the amounts of freezing and nonfreezing water as calculated on the assumption that the specific heat of melting of freezing water is equal to that of pure water, $79.7 \mathrm{cal} \mathrm{g}^{-1}$. The amount of nonfreezing water was determined from the difference in the amounts of total water and freezing water. All water molecules absorbed in the Cuprophan membrane exist as nonfreezing water at a water content lower than about 0.30. The nonfreezing water increases with increasing total water content, approaching about 0.49 asymptotically. Only the freezing water increases at a water content higher than about 1.0. It has also been reported that both nonfreezing and freezing water increase with increasing water content for other cellulosic membranes. ${ }^{7,12,15}$ These data may be comparable with those for poly(2-hydroxyethyl methacrylate), which indicate that the freezing water increases only after nonfreezing water has been saturated. ${ }^{19}$

Figure 8 shows the relationship between water content and the heat of melting of freezing water for various membranes. The data points for each membrane, except the bPAN 5 membrane, do not follow a straight line but give a curve convexing downward at lower water content. The slope of each straight part of the curve is approximately equal to the specific heat of melting of pure water, thus validating the assumption mentioned above. Figures 7 and 8 show that the amount of nonfreezing water is not saturated at lower water content and the equilibrium nonfreezing water content, $W_{\text {non }}$, was thus estimated by extrapolation of the straight part of the curves to $\Delta H=0$. The results are summarized in Table II. In some

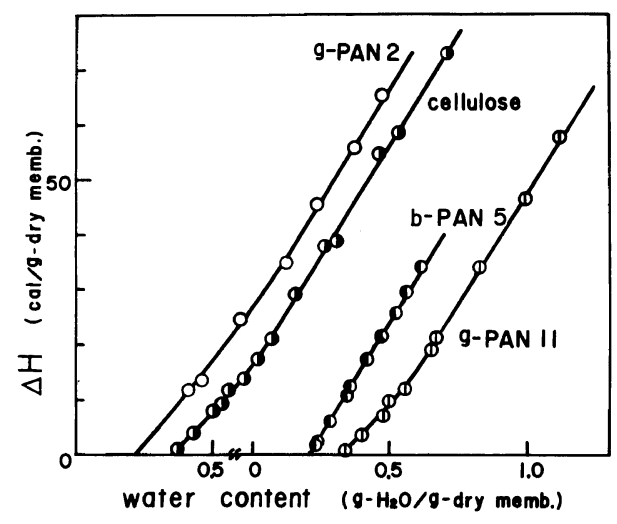

Figure 8. Total water content dependence of the heat of melting of freezing water for various membranes: $(O)$ g-PAN 2; (O) cellulose; (D) b-PAN 5; (D) g-PAN 11.

cases, the freezing water content of membranes is negative since, as mentioned in our previous paper, ${ }^{23}$ the equilibrium water content as determined by blotting water on the membrane surfaces, $W_{\text {blot }}$, may be considerably inaccurate. We thus determined the equilibrium water content by the DSC method, $W_{\mathrm{DSC}}$, and expressed it as the water content that nonfreezing water is saturated, i.e., water content such that the plots of $\Delta H$ against it deviate from the straight line of slope, $79.7 \mathrm{cal} \mathrm{g}^{-1}$. The results obtained are summarized in the third column of Table II. The freezing water content of the g-PAN 2 membrane is found to be higher than that of the cellulose membrane, indicating the superiority of permeability through the g-PAN 2 membrane to that through the cellulose membrane even though the water content of both is the same. Moreover, the water content of the g-PAN 1 membrane is somewhat less than that of the cellulose membrane, but the order of the freezing water content is reversed. The amount of freezing water may be the reason for the somewhat superior permeability through the g-PAN 1 membrane compared to that through the cellulose membrane.

The freezing water content of the Cuprophan membrane is higher than that of the g-PAN 2 membrane. This is inconsistent 
Table II. DSC measurements for cellulosic, grafted, and blended membranes

\begin{tabular}{|c|c|c|c|c|c|}
\hline \multirow{3}{*}{ Sample code } & \multicolumn{2}{|c|}{$\begin{array}{c}\text { Equilibrium } \\
\text { water content }\end{array}$} & \multirow{2}{*}{$\begin{array}{c}\begin{array}{c}\text { Equilibrium nonfreezing } \\
\text { water content }\end{array} \\
W_{\text {non }}\end{array}$} & \multicolumn{2}{|c|}{$\begin{array}{c}\text { Equilibrium freezing } \\
\text { water content }\end{array}$} \\
\hline & $W_{\text {blot }}$ & $W_{\mathrm{DSC}}$ & & $\left(W_{\text {blot }}-W_{\text {non }}\right)$ & $\left(W_{\mathrm{DSC}}-W_{\mathrm{non}}\right)$ \\
\hline & \multicolumn{5}{|c|}{ g- $\mathrm{H}_{2} \mathrm{O} /$ g-dry membrane } \\
\hline \multicolumn{6}{|l|}{ Cellulosic } \\
\hline Cuprophan & 0.96 & 1.00 & 0.49 & 0.47 & 0.51 \\
\hline Cellulose & 0.70 & 0.70 & 0.45 & 0.25 & 0.25 \\
\hline \multicolumn{6}{|l|}{ H-GE Membrane } \\
\hline g-PAN 1 & 0.62 & 0.65 & 0.37 & 0.25 & 0.28 \\
\hline g-PAN 2 & 0.73 & 0.70 & 0.32 & 0.41 & 0.38 \\
\hline \multicolumn{6}{|l|}{ L-GE Membrane } \\
\hline g-PAN 11 & 0.46 & 0.62 & 0.41 & 0.05 & 0.21 \\
\hline g-PAN 12 & 0.29 & 0.40 & 0.30 & -0.01 & 0.10 \\
\hline g-PAN 13 & 0.28 & 0.35 & 0.27 & 0.01 & 0.08 \\
\hline g-PAN 14 & 0.18 & 0.25 & 0.20 & -0.02 & 0.05 \\
\hline \multicolumn{6}{|c|}{ Blended membrane } \\
\hline b-PAN 1 & 0.57 & 0.57 & 0.43 & 0.14 & 0.14 \\
\hline b-PAN 3 & 0.28 & 0.29 & 0.29 & -0.01 & 0 \\
\hline b-PAN 5 & 0.17 & 0.21 & 0.21 & -0.04 & 0 \\
\hline PAN & - & 0.04 & 0.04 & - & 0 \\
\hline
\end{tabular}

Table III. Rough estimation of free, weakly interacting, and strongly interacting water

\begin{tabular}{|c|c|c|c|c|}
\hline \multirow{3}{*}{ Sample code } & \multirow[b]{2}{*}{$\begin{array}{c}\text { Freezing } \\
\text { water }\end{array}$} & \multirow[b]{2}{*}{$\begin{array}{l}\text { Free } \\
\text { water }\end{array}$} & \multicolumn{2}{|c|}{ Intermediate water } \\
\hline & & & $\begin{array}{c}\text { Weakly } \\
\text { interacting }\end{array}$ & $\begin{array}{c}\text { Strongly } \\
\text { interacting }\end{array}$ \\
\hline & \multicolumn{4}{|c|}{ g- $\mathrm{H}_{2} \mathrm{O} /$ g-dry membrane } \\
\hline Cuprophan & 0.51 & 0.24 & 0.14 & 0.13 \\
\hline Cellulose & 0.25 & 0.22 & 0 & 0.03 \\
\hline g-PAN 2 & 0.38 & 0.30 & 0.08 & 0 \\
\hline g-PAN 13 & 0.08 & 0.08 & 0 & 0 \\
\hline b-PAN 3 & 0 & 0 & 0 & 0 \\
\hline
\end{tabular}

with the data in Figure 1, that the permeabilities of uric acid, raffinose, and vitamine $B_{12}$ through the g-PAN 2 membrane are superior to those through the Cuprophan membrane, implying that the freezing water content is not the only measure of solute diffusive permeability.

In Figure 2, the fraction of intermediate water for the Cuprophan membrane is much larger than those of other membranes.
Furthermore, as can be seen from Figure 4, the amount of relatively strongly interacting water for the g-PAN 2 membrane is small. The endotherms for various membranes can be resolved roughly into each type of water and are listed in Table III. The amount of free water for the g-PAN 2 membrane is larger than that for the Cuprophan membrane. The assumption that the intermediate water for the Cuprophan membrane is not appreciably re- 
lated to the diffusive permeation of solutes may explain the good permeability through the g-PAN 2 membrane.

However, the interaction between permeating solutes, membrane substrates, and water in membranes should be considered since, as can be seen from Figure 1, the permeabilities of all solutes investigated through the g-PAN 2 membrane are not necessarily superior to those through the Cuprophan membrane. It has been reported that solutes having water structure breaking hydrophilic groups are closely accessible to polymer chains ${ }^{20,30-32}$ and that the hydraulic permeability of water for an aqueous solution of poly(ethylene glycol) is greater than that for pure water. ${ }^{33}$ Moreover, it has been also reported ${ }^{26,27,34}$ that the water content of membranes swollen in pure water differs from that swollen in solutions and the partition coefficient of solutes varies with the solute. To clarify the interaction between permeating solutes, membrane substrates, and water in membranes, an investigation of the states of water in membranes swollen in solutions is now in progress.

As indicated in Table II, freezing water is nearly absent in the b-PAN 3 and b-PAN 5 membranes, but there is permeation of the solutes through them. ${ }^{23}$ This means that solutes can diffuse through the bound water regions, which is consistent with the previous finding that bound water can be devided into two kinds of water, one containing solutes and one void of solutes. ${ }^{15}$ The equilibrium water content of the b-PAN 3 membrane is $0.28-$ 0.29 and thus the thermograms shown in Figure 6 should correspond to extra surface water, referred to as "bulk water." ${ }^{2,16}$ But the relatively strongly interacting water peaks and the pronounced tailing of free water peaks can be recognized on these thermograms and are comparable with the thermograms of the PAN membrane and pure water, which indicated only sharp free water peaks. It remains unclear whether solutes can diffuse through the bound water regions. At the present, it is only clear that water soluble solutes are difficult to diffuse through intermediate water regions and even more difficult to diffuse through bound water regions.

The equilibrium nonfreezing water content per one gram cellulose for various membranes is in the range of $0.41-0.52 \mathrm{~g}$ and does not depend on the kind of membrane and PAN content. Thus, as shown in Figure 4, grafting with AN onto cellulose has a significant effect not on the nonfreezing water content but intermediate water content.

\section{REFERENCES}

1. D. G. Pedley and J. B. Tighe, Br. Polym. J., 11, 130 (1979).

2. A. Odajima, J. Phys. Soc. Jpn., 14, 308 (1959).

3. R. E. Dehl, J. Chem. Phys., 48, 831 (1968).

4. M. A. Frommer, M. Shporer, and R. M. Messalem, J. Appl. Polym. Sci., 17, 2263 (1973).

5. S. Krishnamurthy, D. McIntyre, E. R. Santee, Jr., and C. W. Wilson, III, J. Polym. Sci., Polym. Phys. Ed., 11, 427 (1973).

6. M. Shporer and M. A. Frommer, J. Macromol. Sci., Phys., B10, 529 (1974).

7. F. C. Magne, H. J. Portas, and H. Wakeham, J. Am. Chem. Soc., 69, 1896 (1968).

8. M. A. Frommer and D. Lancet, J. Appl. Polym. Sci., 16, 1295 (1972).

9. H. G. Burghoff and W. Pusch, J. Appl. Polym. Sci., 23, 473 (1979).

10. M. Aizawa and S. Suzuki, Bull. Chem. Soc. Jpn., 44, 2967 (1971).

11. M. Aizawa, J. Mizuguchi, S. Suzuki, S. Hayashi, T. Suzuki, N. Mitomo, and H. Toyama, Bull. Chem. Soc. Jpn., 45, 3031 (1972).

12. M. F. Froix and R. Nelson, Macromolecules, 8, 726 (1975).

13. H. B. Lee, M. S. Jhon, and J. D. Andrade, J. Colloid Interface Sci., 51, 225 (1975).

14. S. Choi, M. S. Jhon, and J. D. Andrade, J. Colloid Interface Sci., 61, 1 (1977).

15. Y. Taniguchi and S. Horigome, J. Appl. Polym. Sci., 19, 2743 (1975).

16. R. A. Nelson, J. Appl. Polym. Sci., 21, 645 (1977).

17. E. Ahad, J. Appl. Polym. Sci., 22, 1665 (1978).

18. C. Lemoyne, C. Friedrich, J. L. Halary, C. Noël, and L. Monnerie, J. Appl. Polym. Sci., 25, 1883 (1980).

19. Y. K. Sung, D. E. Gregonis, M. S. Jhon, and J. D. Andrade, J. Appl. Polym. Sci., 26, 3719 (1981).

20. S. C. Yoon and M. S. Jhon, J. Appl. Polym. Sci., 27, 3133 (1982). 
21. G. N. Ling, "Water Structure at the Water-Polymer Interface," H. H. G. Jellinek, Ed., Plenum, New York, 1972, p 4.

22. T. Matsuura, "Gouseimaku no Kiso," Kitami Shobo, Tokyo, 1981.

23. N. Nishioka, K. Watase, K. Arimura, K. Kosai, and M. Uno, Polym. J., 16, 867 (1984).

24. S. Krause, "Polymer Blends," Vol. 1, D. R. Paul and S. Newman, Ed., Academic Press, New York, 1978, p 15.

25. N. Nishioka, in preparation.

26. H. Yasuda, C. E. Lamaze, and L. D. Ikenberry, Makromol. Chem., 118, 19 (1968).

27. H. Yasuda, L. D. Ikenberry, and C. E. Lamaze, Makromol. Chem., 125, 108 (1969).
28. H. Yasuda, A. Peterlin, C. K. Colton, K. A. Smith, and E. W. Merrill, Makromol. Chem., 126, 177 (1969).

29. G. Ceccorulli, M. Scandola, and G. Pezzin, Biopolymers, 16, 1505 (1977).

30. E. G. Finer, F. Franks, and M. J. Tait, J. Am. Chem. Soc., 94, 4424 (1972).

31. O. D. Bonner, J. M. Badnarek, and R. K. Arisman, J. Am. Chem. Soc., 99, 2898 (1977).

32. S. J. Wisniewski and S. W. Kim, J. Membrane Sci., 6, 299 (1980).

33. T. Uragami, Y. Sugitani, and M. Sugihara, Polymer, 23, 192 (1982).

34. S. Takigami, Y. Maeda, and Y. Nakamura, J. Appl. Polym. Sci., 24, 1419 (1979). 